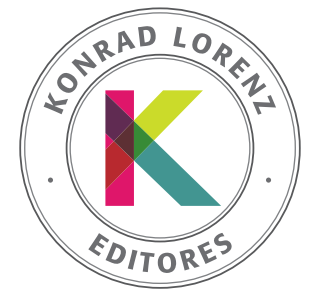

SUMA PSICOLÓGICA

http://editorial.konradlorenz.edu.co/suma-psicologica.html

\title{
Desarrollo y validación de una escala para medir satisfacción con los recursos laborales
}

\author{
Carlos L. Spontóna, Mario A. Trógolo ${ }^{\mathrm{a}, \mathrm{b}}$, Estanislao Castellano \\ Luis P. Morera ${ }^{\mathrm{a}}$ y Leonardo Medrano ${ }^{\mathrm{a}, \mathrm{c}}$
}

a Universidad Siglo 21 (US21), Córdoba, Argentina

${ }^{b}$ Universidad Nacional de Córdoba (UNC), Córdoba, Argentina

c Universidad Católica Madre y Maestra, Escuela de Psicología, República Dominicana

Recibido el 11 de abril de 2019; aceptado el 3 de julio de 2019

PALABRAS CLAVE

Recursos laborales, satisfacción, desarrollo y validación, medición

\begin{abstract}
Resumen En los últimos 30 años la investigación empírica ha mostrado consistentemente la influencia positiva de los recursos laborales en el bienestar y desempeño de los trabajadores. Sin embargo, se ha señalado que estos resultados positivos se asocian más con la satisfacción percibida que con los recursos provistos por la organización. En esta línea y tomando como referencia el modelo HERO se diseñó un instrumento para medir la satisfacción con los recursos laborales. Los análisis factoriales exploratorio y confirmatorio utilizando muestras de 492 y 508 trabajadores, respectivamente, respaldaron las cuatro dimensiones teóricas propuestas: satisfacción con los recursos de tarea, satisfacción con los recursos de equipo, satisfacción con los recursos de líder y satisfacción con los recursos de la organización. Se obtuvieron índices satisfactorios de consistencia interna y de fiabilidad del constructo, y evidencias de validez test-criterio con medidas de engagement y burnout. Se discute el valor práctico del nuevo instrumento y algunas sugerencias tendientes a examinar en mayor profundidad sus propiedades psicométricas.
\end{abstract}

(c) 2019 Fundación Universitaria Konrad Lorenz. Este es un artículo Open Access bajo la licencia CC BY-NC-ND (http://creativecommons.org/licenses/bync-nd/4.0/).

\section{Development and validation of a measure of satisfaction with job resources}

\section{KEYWORDS}

Job resources, satisfaction, development and validation, measurement
Abstract Over the past three decades research on job resources has consistently shown their influence on employees' well-being and performance. However, recent studies indicate that such outcomes are more strongly related to satisfaction with job resources than to perceived job resources. Accordingly, and based on HERO model, we developed a new measure to assess satisfaction with job resources. Exploratory and confirmatory factor analytic-results based on two independent samples consisting of 492 and 508 employees supported the four proposed dimensions: satisfaction with task resources, satisfaction with team resources, satis-

* Autor para correspondencia.

Correo electrónico: mario.trogolo@gmail.com, mario.trogolo@ues21.edu.ar 
faction with leader/supervisor resources, and satisfaction with organizational resources. Reliability analyses showed good internal consistency and construct reliability for the dimensions, and relations with burnout and work engagement scales supported for criterion validity. Practical implications of the new measure are discussed, and we also made some suggestions to examine the psychometric properties of the scale in a more substantial degree.

(c) 2019 Fundación Universitaria Konrad Lorenz. This is an open access article under the CC BYNC-ND license (http://creativecommons.org/licenses/bync-nd/4.0/).

En la actualidad existen constructos teóricos e instrumentos que permiten conocer, explicar y medir las principales variables asociadas al bienestar y la motivación de las personas en el trabajo y sus niveles de salud ocupacional. En este marco, surge la psicología organizacional positiva con el objetivo de describir, explicar y predecir el funcionamiento óptimo en estos contextos, así como optimizar y potenciar la calidad de vida laboral y organizacional (Salanova, Martínez \& Llorens, 2014; Salanova \& Schaufeli, 2004). Este enfoque busca brindar respuestas a las necesidades emergentes que han surgido debido a los cambios culturales, sociales y tecnológicos (Stacey et al., 2018), como así también a los cambios en la organización del trabajo (Llorens, Salanova \& Ventura, 2009; Schaufeli, Leiter \& Maslach, 2009).

Dentro de este contexto, unos de los desarrollos teóricos más importantes es el concepto de Organizaciones Saludables. Éstas son entendidas como aquellas organizaciones que desarrollan prácticas saludables de estructurar y gestionar los procesos de trabajo que influyen en el desarrollo de empleados saludables; y tienen outputs saludables, es decir, resultados que favorecen la supervivencia y el desarrollo de esa organización a través del logro de sus objetivos (Salanova, 2008). En esta línea de trabajo, Salanova, Llorens, Cifre y Martínez (2012) propusieron el modelo HERO (Healthy and Resilient Organization) para abordar integralmente a las personas, grupos y organizaciones. Este modelo entiende que las prácticas y recursos de la organización aportan bienestar y favorecen el desarrollo de entornos saludables de trabajo.

Por su parte, Bakker y Demerouti (2018) desarrollaron la teoría de Demandas y Recursos Laborales en la cual se definen los recursos laborales como aquellos aspectos físicos, psicológicos, organizacionales o sociales del trabajo que: (a) reducen las exigencias del trabajo y los costos fisiológicos y psicológicos asociados, (b) son decisivos en la consecución de los objetivos del trabajo, y (c) estimulan el crecimiento personal, el aprendizaje y el desarrollo.

Desde que se empezaron a estudiar los recursos laborales, coexisten diferentes clasificaciones respecto a la cantidad, tipos e impactos sobre la motivación y el bienestar. Una de las primeras clasificaciones fue propuesta por Warr (1990) quien planteó un modelo de nueve recursos laborales (conocido como modelo "vitamínico") relacionados con las tareas del trabajador o con su entorno social inmediato: claridad de rol laboral, autonomía, oportunidades para el contacto social, variedad de las tareas, feedback sobre el trabajo, salario justo, seguridad física, valoración social y apoyo del supervisor. En una clasificación más integral, Salanova (2008) propuso dos tipos de recursos que la empresa u organización puede proveer al trabajador para cuidar su bienestar y facilitar su desempeño: (1) recursos estructurales, los cuales refieren al ambiente de trabajo e incluyen los recursos de tarea y los recursos de la organización, y (2) recursos sociales, que refieren a las interacciones sociales con las personas en el trabajo (jefes, compañeros, colaboradores, clientes, etc.)

En el presente trabajo proponemos conceptualizar los recursos laborales sobre la base de cuatro dimensiones: (a) recursos de tarea, (b) recursos de equipo, (c) recursos del líder, y (d) recursos de la organización. Esta clasificación parte del modelo HERO (Salanova, Llorens \& Martínez, 2019) pero diferencia los recursos interpersonales en dos dimensiones para lograr mayor especificidad. Para ello ubica por un lado a los factores motivadores que puedan surgir de las interacciones con el grupo o equipo de trabajo, denominados aquí como recursos de equipo; y por otro lado a los factores estimulantes que pueda recibir el trabajador a partir de la relación con quienes lo lideran (jefe, supervisor inmediato), es decir, los recursos del líder. Esta distinción se basa en el hecho de que los trabajadores establecen relaciones interpersonales diferentes con las figuras de autoridad, supervisores, trabajadores de mayor jerarquía en el organigrama y líderes formales o informales, y con sus pares y compañeros de trabajo. La diferencia principal radica en la asimetría de poder propia de las relaciones jerárquicas, donde el proceso de influencia interpersonal condiciona el comportamiento de la persona liderada (Yukl, Gordon \& Taber, 2002). Asimismo, diferentes estudios sugieren un impacto diferente de las relaciones con el líder, y con los pares y compañeros en el bienestar de los empleados (Breevaart \& Bakker, 2018; Thompson \& Prottas, 2005). Por ejemplo, Schaufeli, Taris y van Rhenen (2008) hallaron que el apoyo del supervisor se asociaba negativamente con burnout, mientras que el apoyo de los compañeros no presentaba ninguna relación. A su vez, el apoyo de los compañeros se relacionaba con mayores niveles de engagement en los empleados, mientras que el apoyo de los supervisores no tenía ningún efecto.

La importancia de la relación con el líder para la motivación, el bienestar y el desempeño ha sido uno de los factores más destacados en los estudios dentro de la psicología y sociología (Tripiana \& Llorens, 2015; Trógolo, Pereyra \& Spontón, 2013). Así, dada la influencia del líder sobre la persona, según lo expresado arriba, lo consideramos como un recurso motivacional distinto de aquellos procesos interpersonales que surgen en dinámicas interaccionales más horizontales con compañeros de trabajo (Cannon-Bowers \& Bowers, 2010; Sánchez-Manzanares, Rico \& Gil, 2008).

Los recursos laborales constituyen un factor de importancia para el bienestar y el desempeño; sin embargo, es importante señalar la diferencia que existe entre los recursos laborales que brinda la organización y la satisfacción que tienen los trabajadores sobre éstos. En efecto, dos personas que trabajan en el mismo puesto, dotadas con los 
mismos recursos laborales, pueden presentar distinto grado de satisfacción (Lent \& Brown, 2008). Diferentes estudios concuerdan en señalar que los niveles de bienestar se explican más sobre los juicios de satisfacción y apoyo percibido que por los recursos provistos por la organización (Duffy \& Lent, 2009).

Se hace necesario, por lo tanto, disponer de medidas específicas orientadas a la evaluación de la satisfacción con diferentes recursos laborales. Vale la pena destacar que existen instrumentos que evalúan recursos laborales, como el cuestionario RED (Salanova, Llorens, Cifré \& Martinez, 2006) o medidas basadas en el modelo HERO (Salanova et al., 2019). Sin embargo, no hemos encontrado en la literatura instrumentos que midan la satisfacción percibida con los recursos laborales en general y desde la propuesta teórica que sustenta el presente trabajo en particular (Tabla 1). En función de ello, el presente trabajo tiene como objetivo desarrollar y validar un instrumento para evaluar la satisfacción con los recursos laborales de los trabajadores. Para ello se realizó lo siguiente: (1) se desarrollaron ítems, (2) se realizó una evaluación de la calidad de los mismos en base al criterio de expertos, (3) se efectuó un examen preliminar de los datos, (4) se llevaron a cabo análisis de validez de estructura interna y de confiabilidad (consistencia interna y fiabilidad del constructo), y (5) se obtuvieron evidencias de validez test-criterio con medidas de engagement y burnout. Si bien no hay antecedentes específicos que examinen la relación entre estos constructos, estudios previos que analizan la satisfacción laboral muestran relaciones positivas y negativas entre este constructo y el engagement y burnout, respectivamente (e.g., Durán, Extremera, Montalbán \& Rey, 2005; Rothmann, 2008). Asimismo, considerando el carácter motivacional de los recursos laborales (Salanova 2008), se plantea como hipótesis que la satisfacción con los recursos laborales tendrá una influencia positiva sobre el engagement y un impacto negativo en el burnout.

\section{Método}

\section{Participantes}

Se utilizó una muestra compuesta por 1000 trabajadores argentinos seleccionados a partir de un muestreo aleatorio telefónico (Hernández Sampieri, Fernández Collado \& Baptista Lucio, 2010). Para ello se establecieron en primer lugar las áreas geográficas que iban a ser muestradas y sus correspondientes códigos telefónicos. Luego, los números a marcar se generaron al azar hasta cumplir con los requerimientos de la muestra. El trabajo de campo fue realizado por cinco encuestadores telefónicos con experiencia, que fueron previamente instruidos. La recolección de datos se realizó entre los meses de mayo y julio de 2017. Los encuestados eran elegibles si: (1) tenían al menos 18 años de edad, (2) vivían en el domicilio, y (3) trabajan en relación de dependencia al momento del estudio. Cabe destacar que la tasa de respuesta a las llamadas telefónicas fue alta (94\%). Todos los participantes fueron debidamente informados sobre los objetivos del estudio y no recibieron ningún tipo de compensación. En la Tabla 2 se describen las características de la muestra.

\section{Instrumentos}

Satisfacción con los recursos laborales. Tomando como marco de referencia el modelo HERO propuesto por Salanova et al. (2012), se diseñó un cuestionario de 16 ítems con el propósito de medir la satisfacción con diferentes recursos laborales (Cuestionario de Satisfacción con los Recursos Laborales; CSRL_16): Recursos del Líder (4 ítems), Recursos de Tarea (4 ítems), Recursos de Equipo (4 ítems), y Recursos Organizacionales (4 ítems). Todos los ítems se responden en una escala Likert de cinco categorías: (1) totalmente en desacuerdo, (2) en desacuerdo, (3) ni en desacuerdo ni de acuerdo, (4) de acuerdo, y (5) totalmente de acuerdo.

Work engagement. Se aplicó la Utrech Work Engagement Scale (UWES; Schaufeli, Salanova, González-Romá \& Bakker, 2002) conformada por 17 ítems que evalúa las tres dimensiones teóricas del engagement: vigor (6 ítems), dedicación (6 ítems), y absorción (5 ítems). Todos los ítems se responden en una escala de Likert con siete opciones, desde 0 (nunca) a 6 (siempre o casi siempre). Estudios realizados evidencian buenas propiedades psicométricas del cuestionario UWES en la población Argentina (Spontón, Medrano, Maffei, Spontón \& Castellano, 2012), confirmándose la estructura tridimensional de la escala junto con índices de consistencia interna satisfactorios en todas las dimensiones. En el presente estudio, los índices de fiabilidad (alfa ordinal) en cada una de las escalas fueron: vigor = .76 , dedicación $=.83$ y absorción $=.77$.

Burnout. Se evaluó mediante el Maslach Burnout Inventory-General Survey (MBI-GS; Schaufeli, Leiter, Maslach \& Jackson, 1996). La escala original consta de 16 ítems y tres factores: agotamiento (5 ítems), cinismo (5 ítems) e ineficacia profesional (6 ítems). A pesar de ello, el agotamiento y el cinismo se consideran las dimensiones centrales del burnout (Halbesleben \& Demerouti, 2005). En Argentina, estudios mediante análisis factorial confirmatorio mostraron que un modelo bifactorial representado por agotamiento y cinismo fue el que mejor ajuste presenta en los datos (Spontón, Trógolo, Medrano \& Castellano, 2019). Por lo tanto, en este trabajo se utilizaron solamente las escalas de agotamiento y cinismo del MBI-GS. En la presente muestra, la fiabilidad obtenida (coeficiente alfa ordinal) para las escalas de agotamiento y cinismo fue .77 y .75 , respectivamente.

Cuestionario socio-demográfico. Se elaboró un cuestionario ad hoc a través del cual se recogió información relativa al sexo, edad, puesto de trabajo, sector que pertenece la organización/empresa y nivel educativo de los trabajadores, entre otros aspectos.

\section{Procedimiento}

Para la elaboración del instrumento se tuvieron en cuenta las directrices establecidas para la construcción y desarrollo de test psicológicos (Eignor, 2013). La primera fase incluyó la delimitación semántica del constructo y el desarrollo de ítems a partir de la revisión bibliográfica, la experiencia profesional de los autores en el contexto organizacional y la realización de dos focus group con trabajadores y profesionales. En la construcción de los ítems se tuvo especial cuidado en desarrollar ítems con un lenguaje accesible a la población meta, y en evitar ítems muy largos, redacción ambigua y uso de expresiones extremas 
Tabla 1 Tabla de especificaciones del instrumento

\section{Constructo a evaluar: satisfacción con los recursos laborales}

Instrumento

Cuestionario de Satisfacción con los recursos laborales (CSRL_16i)

Definición del constructo:

La satisfacción con los recursos laborales comprende el bienestar de las personas en relación a diferentes factores presentes en el contexto laboral, que favorecen/facilitan el desarrollo de la tarea y el logro de los objetivos del trabajo, estimulan el desempeño individual y colectivo, favorecen el desarrollo y crecimiento personal, y promueven/propician ambientes laborales positivos. Estos factores, o recursos, pueden localizarse a distintos niveles:

Líder: abarca aspectos de la relación con el jefe o supervisor, como la claridad de la información que brinda, la retroalimentación y el reconocimiento por las tareas realizadas.

Tarea: se centra en las características intrínsecas o inmediatas del puesto de trabajo, como la disponibilidad de tiempo y de recursos materiales necesarios para realizar las tareas, y la posibilidad de hacer uso de las habilidades y sentirse desafiado por el trabajo que se realiza.

Equipo: engloba el ambiente socio-laboral, particularmente la relación con los compañeros o con el equipo de trabajo en aspectos ligados a la cooperación, coordinación, productividad o eficiencia, y la creatividad en la resolución de problemas.

Organización: se refieren a las condiciones laborales y las prácticas organizacionales en un sentido general. Incluye aspectos como el salario, sistema de recompensas no económicas, beneficios, oportunidades de desarrollo, capacitación y aprendizaje.

\section{Población diana}

El test se encuentra dirigido a personas en general que trabajan en empresas y organizaciones de diferentes rubros (industrial, comercial, servicios, agrónomo) y sectores (público, privado y organizaciones sin fines de lucro). Se pretende crear un test genérico que pueda aplicarse en estos ámbitos, independientemente de las tareas específicas que desarrollen los trabajadores.

\section{Objetivos de aplicación}

El test fue creado con propósitos de ser usado tanto en investigación como en la práctica profesional. Se pretende que el cuestionario sea una herramienta de evaluación útil sobre la satisfacción con los distintos recursos en el trabajo (a nivel individual, del líder, grupal u organizacional) que pueda ser usado por profesionales, consultores organizaciones y responsables de gestión de recursos humanos para orientar el diseño de intervenciones y evaluar el impacto de mejoras en los recursos laborales.

\section{Aspectos operativos relacionados con la aplicación del instrumento}

Se busca disponer de un instrumento breve que pueda ser respondido en 5 minutos, de forma que pueda aplicarse conjuntamente con otras medidas.

Tabla 2 Características socio-demográficas y laborales de la muestra

\begin{tabular}{|c|c|c|c|c|c|}
\hline & $\mathbf{N}$ & $\%$ & & $\mathbf{N}$ & $\%$ \\
\hline Sexo & & & \multicolumn{3}{|l|}{ Sector en el que trabaja } \\
\hline Hombre & 485 & 48.5 & Público & 304 & 30.4 \\
\hline \multirow[t]{2}{*}{ Mujer } & 515 & 51.5 & Privado & 653 & 65.3 \\
\hline & & & Tercer Sector (ONGs) & 29 & 2.9 \\
\hline \multicolumn{3}{|l|}{ Edad } & $\mathrm{Ns} / \mathrm{Nc}$ & 14 & 1.4 \\
\hline $18-30$ años & 218 & 21.8 & & & \\
\hline 31-40 años & 269 & 26,9 & \multicolumn{3}{|c|}{ Rubro al que pertenece la organización/empresa } \\
\hline 41-50 años & 257 & 25.7 & Industrial & 110 & 11 \\
\hline \multirow[t]{2}{*}{$51-65$ años } & 256 & 25.6 & Comercial & 200 & 20 \\
\hline & & & Servicios & 681 & 68.1 \\
\hline \multicolumn{3}{|l|}{ Nivel de estudios } & Agro & 8 & 0.8 \\
\hline Primario incompleto & 10 & 1 & $\mathrm{Ns} / \mathrm{Nc}$ & 1 & 0.1 \\
\hline Primario completo & 44 & 4.4 & & & \\
\hline Secundario incompleto & 63 & 6.3 & \multicolumn{3}{|l|}{ Tamaño de la organización/empresa } \\
\hline Secundario completo & 218 & 22 & Microempresa ( $0-10$ personas) & 414 & 41.4 \\
\hline Terciario incompleto & 70 & 7 & Pequeña empresa (10-50 personas) & 225 & 22.5 \\
\hline Terciario completo & 142 & 14.3 & Mediana empresa (50-250 personas) & 135 & 13.5 \\
\hline Universitario incompleto & 167 & 16.8 & Empresa grande (> 250 personas) & 205 & 20.5 \\
\hline Universitario completo & 213 & 21.5 & $\mathrm{Ns} / \mathrm{Nc}$ & 21 & 2.1 \\
\hline Posgrado incompleto (Maestría, Doctorado, Especialidad) & 6 & 0.6 & & & \\
\hline Posgrado completo (Maestría, Doctorado, Especialidad) & 60 & 6 & & & \\
\hline $\mathrm{Ns} / \mathrm{Nc}$ & 7 & 0.7 & & & \\
\hline
\end{tabular}


(Medrano, Pérez \& Fernández, 2019). Mediante este procedimiento se generó un pool inicial de ítems que fue remitido a un panel de expertos compuesto por un especialista en gestión de recursos humanos, un experto en psicología organizacional con conocimientos en psicometría, y un psicólogo laboral. Para la revisión de expertos se creó una tabla de especificaciones de la escala donde se proporcionó información sobre la definición semántica del constructo, sus dimensiones, los objetivos de aplicación del test, la población diana y aspectos operativos relacionados con su uso (ver Tabla 1). Se solicitó a cada uno que evaluara los ítems en cuanto a: (1) pertinencia con el constructo analizado; (2) claridad en la redacción, y (3) relevancia, utilizando una escala de respuesta con 5 opciones (desde 1 = totalmente en desacuerdo, hasta 5 = totalmente de acuerdo)

En base a los ítems que presentaron mayor grado de acuerdo inter-jueces se confeccionó un cuestionario de 16 ítems que fue sometido a un pre-test en una muestra independiente de 100 trabajadores. Como resultado, se efectuaron algunas adecuaciones lingüísticas menores en dos ítems con el objeto de hacerlos comprensibles y evitar confusiones. Con la versión resultante se llevaron a cabo estudios psicométricos tendientes a aportar evidencias de validez y confiabilidad del nuevo instrumento. Tanto en el pre-test como en la muestra definitiva se obtuvo el consentimiento verbal de los participantes una vez que se informó sobre el propósito de la investigación y el carácter anónimo de las respuestas. Esta investigación contó con la aprobación del Comité de Ética en Investigación (CEI) de la universidad en la que se radicó el estudio.

\section{Análisis de datos}

Se evaluó el grado de acuerdo entre los jueces sobre la calidad de los ítems a través del coeficiente $V$ de Aiken (Aiken, 1985) y sus intervalos de confianza respectivos utilizando el programa elaborado por Merino y Livia (2009). Dado que el número de jueces es pequeño, se fijó un nivel de confianza del $90 \%$ y se estableció como criterio para retener el ítem un valor $\mathrm{V} \geq .50$ correspondiente al límite inferior del intervalo (Penfield \& Giacobbi, 2004). A continuación se efectuó un análisis preliminar de la base con el fin de analizar los valores perdidos, identificar la presencia de casos atípicos, evaluar la normalidad, linealidad y ausencia de multicolinealidad entre los ítems, y comprobar la adecuación de los datos para el análisis factorial (Furr, 2011). Seguidamente se evaluó la estructura interna de la escala mediante análisis factorial exploratorio (AFE) y confirmatorio (AFC) en dos muestras independientes $\left(n_{1}=492\right.$; $\left.n_{2}=508\right)$. Para el AFE se usó el programa Factor v10.3.01 y para determinar el número de factores a retener se utilizaron diferentes criterios: el análisis paralelo basado en el análisis factorial de rango mínimo (Timmerman \& LorenzoSeva, 2011), la inspección de la distribución de los residuos tipificados, la raíz media cuadrática residual (RMSR) y el índice gamma $(\mathrm{GFI})$. Estos dos últimos índices de ajuste se basan en la evaluación de las correlaciones residuales y se interpretan como adecuados si GFI $\geq .90$ y RMSR resulta inferior al criterio propuesto por Kelley, $1 / \int \mathrm{N}$ (Lloret-Segura, Ferreres-Traver, Hernández-Baeza \& Tomás-Marco, 2014). En cuanto a los residuos, se espera que la distribución sea simétrica, en torno a 0 , con pocos residuos superiores a |2| para considerar que el modelo es adecuado (Arias, 2008). Además de los criterios estadísticos y de los índices de ajuste, se tuvo en cuenta si los factores estaban bien definidos y poseían una interpretación substantiva de acuerdo con la teoría (Fabrigar, Wegener, MacCallum \& Strahan, 1999; Lloret, Ferreres, Hernández \& Tomás, 2017). Dado el nivel ordinal de los ítems, se analizó la matriz de correlaciones policórica y se aplicó el método de mínimos cuadrados no ponderados (ULS) ya que es el más apropiado para el análisis de las matrices de covarianza originadas a partir de correlaciones policóricas (Hofmman, Stover, de la Iglesia \& Fernández-Liporace, 2013). Para interpretar la solución factorial obtenida se empleó una rotación oblicua (Promin) puesto que se esperaba que los factores de la escala estuvieran intercorrelacionados entre sí, hecho que se verificó posteriormente. Si bien existen varios métodos de rotación oblicua disponibles (e.g., Promax, Oblimin directo, Simplimax), estudios de simulación sugieren que Promin ofrece resultados más precisos (cf. Lorenzo-Seva, 1999)

El AFC se realizó mediante el programa Mplus v6.12. Cómo método de estimación se usó el WLSMV (Weigthed Least Squares Mean and Variance; Li, 2016) y para evaluar el ajuste del modelo se consideraron diferentes índices recomendados en la literatura: el estadístico chi-cuadrado $\left(\chi^{2}\right)$, el índice de Tucker-Lewis (TLI), el índice de ajuste comparativo (CFI), el error cuadrático medio de aproximación (RMSEA), y la ponderada media cuadrática residual (WRMR). Valores $\geq 0.95$ en CFI y TLI indican un ajuste óptimo, y valores $\geq 0.90$ representan un ajuste aceptable. Para el RMSEA valores $\leq 0.05$ se consideran óptimos $y \geq 0.08$ aceptables, y finalmente para WRMR se esperan valores $\leq$ 1.00 (Yu \& Muthen, 2002). Se evaluó la confiabilidad de la escala a través del análisis de la consistencia interna de los ítems (coeficiente alfa ordinal) y la fiabilidad del constructo (coeficiente $H$, Dominguez-Lara, 2016). Finalmente, se calcularon correlaciones bivariadas ( $r$ de Pearson) para el análisis de las relaciones test-criterio.

\section{Resultados}

\section{Focus group}

Se llevaron a cabo dos sesiones focales con grupos de trabajadores y profesionales, con el fin de que compartieran su experiencia laboral y brindaran su punto de vista acerca de los aspectos que consideran necesarios y/o importantes para un buen funcionamiento y satisfacción en el trabajo. Cada sesión duró aproximadamente 45 minutos y se llevó a cabo en un aula destinada a tal efecto en la universidad. Todos los participantes fueron informados al comienzo de los objetivos del encuentro. En general, se aprecia que los trabajadores refieren a la importancia de disponer de tiempo y de los elementos "necesarios" para llevar a cabo sus tareas. Se destaca también la importancia de la relación con el supervisor en lo relativo a la información, apoyo, la confianza, y la autonomía que brinda; al igual que la importancia de un buen clima y de una coordinación efectiva para el logro de objetivos en los equipos de trabajo. Por último, aparecen expresiones que denotan el valor del sentido de pertenencia a la organización/empresa y el poder desarrollar un trabajo motivante en el que perciben que pueden crecer y aprender cosas nuevas. 


\section{Selección de ítems - acuerdo entre expertos}

Se elaboró un pool inicial de 20 ítems. A partir de la evaluación de los jueces, se seleccionaron para cada dimensión los cuatro ítems que evidenciaron un mayor grado de acuerdo inter-jueces (coeficientes $\vee$ más altos). Los resultados se presentan en la Tabla 3.

\section{Examen preliminar de los datos}

No se identificaron valores perdidos en la muestra analizada. Para identificar la presencia de casos atípicos univariados se calcularon los puntajes $z$ de cada ítem, considerándose como tales aquellos cuyos valores se encuentran fuera del rango $z \pm 3$ (Tabachnick \& Fidell, 2001). Se observaron 40 casos atípicos univariados distribuidos en los ítems 3,9 y 11. Además, se identificaron 45 casos atípicos multivariados mediante el estadístico de distancia de Mahalanobis $(p<$ .001). Dado que los valores atípicos pueden afectar las correlaciones entre los ítems y, consecuentemente, el análisis factorial, para estimar su impacto se llevó a cabo un análisis factorial con y sin casos atípicos y se comparó la convergencia de las soluciones factoriales, calculando las correlaciones ( $r$ de Pearson) entre las puntuaciones factoriales luego de rotación (Rivas, 1999). Los resultados indican una elevada congruencia entre los factores (valores $r$ comprendidos entre .99 - 1.00) por lo cual se decidió incluir todos los casos en los análisis posteriores. A continuación, se obtuvieron los índices de asimetría y curtosis para cada ítem (ver Tabla 4). Todos los casos valores se encuentran entre \pm

Tabla 3 Ítems seleccionados para la conformación definitiva del CSRL_16 a partir de la valoración de jueces expertos (V de Aiken)

\begin{tabular}{lcccc}
\hline Dimensión & Ítems & V & $\begin{array}{c}\text { Límite inferior } \\
\text { (IC 90\%) }\end{array}$ & $\begin{array}{c}\text { Límite superior } \\
\text { (IC 90\%) }\end{array}$ \\
\hline SRL & 1 & .85 & .65 & .95 \\
SRL & 2 & .83 & .63 & .93 \\
SRL & 3 & .87 & .68 & .96 \\
SRL & 4 & .87 & .68 & .96 \\
SRT & 5 & .81 & .61 & .91 \\
SRT & 6 & .83 & .63 & .93 \\
SRT & 7 & .79 & .58 & .90 \\
SRT & 8 & .85 & .65 & .95 \\
SER & 9 & .85 & .65 & .95 \\
SER & 10 & .79 & .58 & .90 \\
SER & 11 & .85 & .65 & .95 \\
SER & 12 & .91 & .73 & .97 \\
SRO & 13 & .83 & .63 & .93 \\
SRO & 14 & .89 & .71 & .97 \\
SRO & 15 & .91 & .73 & .97 \\
SRO & 16 & .87 & .68 & .96 \\
\hline
\end{tabular}

Nota. SRL: satisfacción con los recursos del líder. SRT: satisfacción con los recursos de tarea. SRE: satisfacción con los recursos de equipo. SRO: satisfacción con los recursos organizacionales
2, lo que puede interpretarse como evidencia de normalidad univariada según los criterios establecidos por George y Mallery (2010). Finalmente, se comprobó la normalidad multivariada de los datos (Mardia = 48.01; Rodríguez Ayán \& Ruiz Díaz, 2008) y la ausencia de multicolinealidad entre los ítems, ya que no se observaron valores de tolerancia y de inflación de la varianza (VIF) inferiores a .10 y superiores a 10, respectivamente (Pérez \& Medrano, 2010).

\section{Análisis factorial exploratorio}

Tanto el índice de adecuación muestral de KaiserMayer-Olkin (KMO $=.90)$ como el test de esfericidad de Bartlett, $\chi^{2}(120)=7227.4, p<.001$, indican que los ítems se encuentran intercorrelacionados, siendo viable el análisis factorial. El análisis paralelo sugirió la existencia de cuatro factores subyacentes que explican el $53 \%$ de la varianza total, GFI = .99; $\mathrm{RMSR}=0.032$ (Criterio de Kelley = 0.045); índice de simplicidad factorial de Bentler $(S)=.99$. Asimismo, el análisis de los residuos tipificados muestra que los residuos se distribuyen de manera simétrica, en torno a cero $(M=.0001 ; M d n=.0002)$ y todos los ítems poseen residuos pequeños $|2|$ excepto el ítem 7 (3.88). Sin embargo, se decidió conservarlo ya que su presencia no tenía un efecto negativo sobre los índices de fiabilidad del factor. El primer factor explicó el $16 \%$ de la varianza e incluye cuatro ítems que refieren a la satisfacción relacionada con diferentes aspectos de la relación con el jefe inmediato/supervisor (e.g., feedback, reconocimiento). En consecuencia, y en consideración con los supuestos teóricos de partida, se optó por denominar a este factor como Satisfacción con los Recursos del Líder (SRL). El segundo factor explicó el 14\% de la varianza y está compuesto por cuatro ítems que engloban la satisfacción asociada a distintos aspectos vinculados con la tarea o el puesto de trabajo (e.g., tiempo, recursos materiales, desarrollo de habilidades). Por lo tanto, se denominó a este factor como Satisfacción con los Recursos de Tarea (SRT). El tercer factor explicó el $12 \%$ de la varianza y agrupa cuatro ítems que miden la satisfacción vinculada con el equipo de trabajo en aspectos como la coordinación, creatividad y colaboración. Acorde con la teoría de base que guío la elaboración del instrumento, se etiquetó a este factor como Satisfacción con los Recursos de Equipo (SRE). Por último, el cuarto factor explicó el $11 \%$ de la varianza y está integrado por cuatro ítems que evalúan la satisfacción con los recursos proporcionados por la organización (e.g., compensaciones económicas y no económicas). Por consiguiente, y en base a los lineamientos teóricos previos, se denominó al cuarto factor como Satisfacción con los Recursos Organizacionales (SRO). En la Tabla 4 se muestran las cargas factoriales (coeficientes de estructura y coeficientes patrón) de cada ítem. Todos los factores presentan correlaciones positivas entre sí, con valores que oscilan entre .51 y .65 (ver Tabla 7).

\section{Análisis factorial confirmatorio}

Con el objetivo de evaluar diferentes modelos alternativos y determinar cuál es la estructura factorial que proporciona una mejor representación de las dimensiones del CSRL_16, se pusieron a prueba cuatro modelos. En concreto, un modelo unidimensional (M1) que asume la satisfacción 
Tabla 4 Matrices factoriales (configuración y estructura), estadísticos descriptivos y comunalidades de los ítems del CSRL_16

\begin{tabular}{|c|c|c|c|c|c|c|c|c|c|}
\hline \multirow[t]{2}{*}{ Items } & \multicolumn{4}{|c|}{ Coef. de configuración / Coef. de estructura } & \multirow[t]{2}{*}{ M } & \multirow[t]{2}{*}{$\mathrm{DE}$} & \multirow[t]{2}{*}{ Asimetría } & \multirow[t]{2}{*}{ Curtosis } & \multirow[t]{2}{*}{$h^{2}$} \\
\hline & SRL & SRT & SRE & SRO & & & & & \\
\hline 1. & $.78 / .76$ & $-.19 / .25$ & $.18 / .41$ & $-.03 / .45$ & 3.81 & 1.36 & -.877 & -.50 & .68 \\
\hline 2. & $.88 / .85$ & $-.09 / .35$ & $.15 / .46$ & $.09 / .43$ & 3.82 & 1.29 & -.869 & -.35 & .76 \\
\hline 3. & $.91 / .90$ & $.02 / .41$ & $-.10 / .39$ & $.05 / .52$ & 3.60 & 1.42 & -.615 & -.94 & .79 \\
\hline 4. & $.85 / .87$ & $.04 / .42$ & $-.05 / .40$ & $.04 / .48$ & 3.77 & 1.36 & -.835 & -.54 & .77 \\
\hline 5. & $.05 / .41$ & $.86 / .87$ & $.01 / .44$ & $-.04 / .40$ & 4.21 & 1.11 & -1.38 & 1.04 & .66 \\
\hline 6. & $-.02 / .30$ & $.85 / .83$ & $-.03 / .40$ & $.02 / .38$ & 4.36 & 1.08 & -1.49 & 2.40 & .62 \\
\hline 7. & $-.04 / .30$ & $.34 / .49$ & $.16 / .32$ & $.14 / .37$ & 3.98 & 1.21 & -1.01 & .01 & .53 \\
\hline 8. & $.03 / .39$ & $.47 / .61$ & $.23 / .35$ & $-.07 / .32$ & 3.71 & 1.35 & -.72 & -.70 & .61 \\
\hline 9. & $.03 / .45$ & -.19 / .31 & $.93 / .84$ & $.01 / .43$ & 3.99 & 1.14 & -1.02 & .36 & .68 \\
\hline 10. & $-.02 / .38$ & $.07 / .47$ & $.90 / .87$ & $-.08 / .42$ & 3.80 & 1.20 & -.83 & -.14 & .72 \\
\hline 11. & $.04 / .43$ & $.12 / .40$ & $.67 / .81$ & $.06 / .38$ & 3.92 & 1.14 & -.98 & .22 & .75 \\
\hline 12. & $-.05 / .39$ & $.09 / .46$ & $.73 / .80$ & $.07 / .46$ & 3.96 & 1.05 & -.99 & .58 & .71 \\
\hline 13. & $-.06 / .36$ & $-.06 / .42$ & $-.01 / .38$ & $.87 / .78$ & 3.46 & 1.40 & -.47 & -1.03 & .67 \\
\hline 14. & $.00 / .43$ & $.36 / .51$ & $.01 / .46$ & $.53 / .75$ & 4.18 & 1.16 & -1.39 & 1.01 & .77 \\
\hline 15. & $.31 / .41$ & $.10 / .47$ & $-.06 / .34$ & $.55 / .68$ & 3.79 & 1.34 & -.84 & -.49 & .81 \\
\hline 16. & $-.13 / .44$ & $.01 / .48$ & $.13 / .32$ & $.96 / .93$ & 3.25 & 1.38 & -.25 & -1.14 & .65 \\
\hline
\end{tabular}

con los recursos laborales como un constructo unitario; un modelo de tres factores correlacionados (M2) representados por "satisfacción con los recursos de tarea", "satisfacción con los recursos sociales" (que incluye los ítems referidos a la satisfacción con los recursos del líder y la satisfacción con los recursos del equipo) y "satisfacción con los recursos organizacionales". Este modelo se desprende de la clasificación propuesta por el modelo HERO (Salanova et al., 2019). El tercer modelo (M3) está compuesto por cuatro factores que resultan de nuestro análisis exploratorio previo (ver Tabla 4). Finalmente, el cuarto modelo (M4) es un modelo jerárquico con dos factores de segundo orden, que representan la satisfacción con los "recursos estructurales" (incluye los recursos de tarea y los recursos organizacionales como factores de primer orden) y la satisfacción con los "recursos sociales" (incluye los recursos del equipo y los recursos del líder como factores de primer orden); y se fundamenta en la diferenciación previa propuesta por Salanova (2008). Los resultados indican que el modelo unidimensional y el modelo de tres factores correlacionados no presentan un buen ajuste en los datos. En cambio, el modelo de cuatro factores surgido del AFE y el modelo jerárquico evidencian índices de ajuste satisfactorios y similares entre sí (Tabla 5). De esta manera, conforme a lo que hemos planteado en este trabajo, la satisfacción con los recursos laborales pueden ser conceptualizada a partir de cuatro dimensiones (líder, equipo, tarea, organizacionales). A su vez, estas cuatro dimensiones pueden ser integradas dentro de dos

Tabla 5 Índices de ajuste correspondientes a los diferentes modelos estructurales analizados

\begin{tabular}{llcccccc}
\hline & Descripción & $\chi^{2}$ & gl & CFI & TLI & RMSEA [90\% IC] & WRMR \\
\hline M1 & Unidimensional & $2676.307^{* * *}$ & 104 & .86 & .84 & $.151[.146, .156]$ & 3.50 \\
$M 2$ & Tres factores & $2061.699^{* * *}$ & 101 & .89 & .87 & $.122[.116, .128]$ & 3.02 \\
$M 3$ & Cuatro factores & $831.162^{* * *}$ & 97 & .96 & .95 & $.074[.069, .079]$ & 1.67 \\
$M 4$ & Jerárquico & $852.735^{* * *}$ & 98 & .96 & .95 & $.075[.070, .080]$ & 1.72 \\
\hline
\end{tabular}

Nota: gl: grados de libertad; CFI: índice de ajuste comparativo; TLI: índice de Tucker-Lewis; RMSEA: error cuadrático medio de aproximación; WRMR: ponderada media cuadrática residual. $M 1$ = modelo unidimensional; $M 2$ = modelo de tres factores correlacionados (satisfacción con los recursos de tarea, satisfacción con los recursos sociales y satisfacción con los recursos organizacionales); $M 3$ = modelo de cuatro factores correlacionados (satisfacción con los recursos del líder, satisfacción con losrecursos de tarea, satisfacción con los recursos deequipoysatisfacción con los recursos organizacionales); $M 4$ = modelo jerárquico con dos factores de segundo orden, recursos estructurales (con recursos de tarea y recursos organizacionales como factores de primer orden) y recursos sociales (con recursos del equipo y del líder como factores de primer orden) ${ }^{* * *} p<.001$ 
factores más generales representados por satisfacción con los recursos estructurales y con los recursos sociales, como sugiere Salanova (2008). Sin embargo, dado que el modelo compuesto por cuatro factores confiere mayor especificidad en la evaluación de la satisfacción con los recursos en el trabajo -lo que resulta de mayor interés a los fines prácticos- se optó por trabajar con este modelo. En la Tabla 6 se presenta la solución factorial estandarizada.

\section{Evidencias de confiabilidad}

Para examinar la consistencia interna de cada subescala se calculó el coeficiente alfa ordinal, obteniéndose los siguientes valores: Satisfacción con los Recursos del Líder $\alpha=$ .91, Satisfacción con los Recursos de Tarea $\alpha=.71$, Satisfacción con los Recursos de Equipo $\alpha=.88$, y Satisfacción con los Recursos Organizacionales $\alpha=.81$. En tanto, la estimación de la confiabilidad del constructo evidenció valores satisfactorios en todas dimensiones: $H=.94$ (Satisfacción con los Recursos del Líder), .81 (Satisfacción con los Recursos de Tarea), .91 (Satisfacción con los Recursos del Equipo), y .89 (Satisfacción con los Recursos Organizacionales)

\section{Validez test-criterio}

Se correlacionaron los puntajes de las distintas dimensiones del CSRL_16 con medidas de engagement y burnout. En base a los planteamientos teóricos del modelo HERO (Salanova et al., 2012) y los antecedentes empíricos (Schaufeli \& Taris, 2014), se esperaba que los trabajadores que experimentan un mayor grado de satisfacción con los recursos laborales presentaran puntajes más altos en las escalas de engagement y más bajos en las escalas de burnout. Asimismo, dado el carácter motivacional de los recursos laborales, se hipotetizó que las relaciones serían más fuertes con las escalas de engagement. Los resultados obtenidos confirman las relaciones esperadas entre las distintas escalas (Tabla 6).

\section{Discusión}

El objetivo del presente estudio consistió en desarrollar y validar un instrumento para la evaluación de la satisfacción con los recursos laborales. Para ello se elaboraron y seleccionaron ítems a partir de la evaluación de expertos,

Tabla 6 Saturaciones estandarizadas de los ítems del Cuestionario de Satisfacción con los Recursos Laborales (CSRL_16)

Factor 1. Recursos del Líder

1. Estoy satisfecho con la claridad de las instrucciones que recibo de mi jefe inmediato/ supervisor

2. Estoy satisfecho con el feedback (información sobre mi trabajo) que recibo de mi jefe inmediato/ supervisor

3. Estoy satisfecho con el reconocimiento (aprobación, valorización, elogios, etc.) que recibo de mi jefe inmediato/ supervisor, por mi esfuerzo

4. Estoy satisfecho con el reconocimiento (aprobación, valorización, elogios, etc.) que recibo de mi jefe inmediato/ supervisor, por los resultados de mi trabajo

Factor 2 Recursos de Tarea

5. Estoy satisfecho con el nivel de desafío que representan las tareas que realizo

6. Estoy satisfecho por realizar tareas que aumentan mis habilidades

7. Estoy satisfecho por tener el tiempo necesario para realizar mis tareas

8. Estoy satisfecho con los recursos materiales que tengo para hacer mis tareas

Factor 3: Recursos de Equipo

9. Estoy satisfecho con la colaboración lograda en los grupos/ equipos de trabajo en los que participo

10. Estoy satisfecho con el nivel de creatividad alcanzado en los grupos/ equipos de trabajo en los que participo

11. Estoy satisfecho con la coordinación lograda en los proyectos y tareas realizadas

12. Estoy satisfecho con el nivel de eficiencia (productividad, resultados, cuidado de los recursos) alcanzado en los grupos/ equipos de trabajo en los que participo

Factor 4. Recursos Organizacionales

13. Estoy satisfecho con las compensaciones no económicas y beneficios (capacitación y aprendizaje, flexibilidad horaria, trabajar desde la casa, prestaciones de salud, viajes) que recibo de la organización/ empresa

14. Estoy orgulloso por pertenecer a esta organización/ empresa

15. Me siento valorado por la organización/empresa en la que trabajo 
Tabla 7 Correlación entre las dimensiones del CSRL_16 y medidas de engagement y burnout

\begin{tabular}{|c|c|c|c|c|c|c|c|c|c|}
\hline & 1 & 2 & 3 & 4 & 5 & 6 & 7 & 8 & 9 \\
\hline 1. Satisfacción con los recursos del líder & - & & & & & & & & \\
\hline 2. Satisfacción con los recursos de tarea & $.51^{\star \star *}$ & - & & & & & & & \\
\hline 3. Satisfacción con los recursos de equipo & $.50^{* *}$ & $.57^{* *}$ & - & & & & & & \\
\hline 4. Satisfacción con los recursos organizacionales & $.65^{\star *}$ & $.59^{* *}$ & $.54^{* *}$ & - & & & & & \\
\hline 5. Dedicación & $.25^{\star *}$ & $.41^{* \star}$ & $.31^{\star \star}$ & $.36^{* *}$ & - & & & & \\
\hline 6. Vigor & $.19^{* *}$ & $.29^{* *}$ & $.24^{* *}$ & $.26^{* *}$ & $.61^{\text {*n }}$ & - & & & \\
\hline 7. Absorción & $.20^{\star * *}$ & $.37^{* *}$ & $.26^{* *}$ & $.25^{\star *}$ & $.68^{* *}$ & $.54^{\star *}$ & - & & \\
\hline 8. Agotamiento & $-.13^{* *}$ & -.04 & $-.10^{* *}$ & $-.12^{* \star}$ & .03 & .05 & $.08^{*}$ & - & \\
\hline 9. Cinismo & $-.20^{* *}$ & $-.18^{* *}$ & $-.17^{* *}$ & $-.25^{\star \star}$ & $-.28^{\star \star *}$ & $-.17^{\star \star}$ & $-.16^{* *}$ & $.26^{* *}$ & - \\
\hline
\end{tabular}

${ }^{* *} p<.01 .{ }^{*} p<.05$

y se examinaron propiedades psicométricas relevantes del instrumento en una muestra amplia de trabajadores de la población Argentina. Los resultados obtenidos indican que el CSRL_16 posee adecuada validez de contenido y de constructo. En concreto, el AFE evidenció cuatro factores bien definidos que recogen la satisfacción con los recursos laborales en cuatro niveles: recursos de tarea, recursos del líder, recursos de equipo y recursos organizacionales. Estos resultados fueron posteriormente replicados mediante AFC en una muestra independiente, garantizando así la robustez de la estructura factorial obtenida. Por otra parte, la consistencia interna de cada dimensión fue satisfactoria, con valores que oscilaron entre .71 y .91 , y se obtuvo también evidencia que respalda la fiabilidad del constructo. Finalmente, las correlaciones obtenidas entre las dimensiones del CSRL_16 y las escalas de engagement y burnout fueron consistentes con lo esperado de acuerdo con estudios previos (Durán et al., 2005; Rothmann, 2008), obteniéndose así evidencia complementaria de validez test-criterio. En síntesis, los resultados obtenidos son satisfactorios y demuestran buenas propiedades psicométricas del nuevo instrumento, permitiendo disponer de una herramienta de medición válida para realizar diagnósticos sobre la satisfacción con los recursos, como así también para desarrollar nuevos estudios sobre las personas en el trabajo, favoreciendo las posibilidades de realizar intervenciones que tiendan a mejorar los niveles de bienestar y desempeño.

Tomando esto en consideración el instrumento se propone como una herramienta de utilidad para los profesionales de la salud ocupacional, consultores de organizaciones y responsables del área de recursos humanos, ya que permite conocer de modo fácil, rápido y confiable la satisfacción con los recursos a nivel individual, del líder, grupal u organizacional. Aportando de esta manera información valiosa para el diseño de intervenciones y prácticas organizacionales saludables que favorezcan el desarrollo de empleados y ambientes saludables de trabajo (Bakker \& Demerouti, 2013). Además, se puede utilizar este instrumento para medir el impacto en las mejoras de los recursos laborales, lo que permite potenciar y optimizar los niveles de bienestar y desempeño. Al contar con datos confiables, se pueden desarrollar acciones de mejora y optimización del trabajo mediante tres focos: (1) potenciando los recursos relaciona- dos con la tarea, (2) implantando nuevos recursos laborales, y (3) mejorando los recursos sociales del lugar de trabajo (Salanova, 2008). En este caso, el CSRL_16 ofrece la ventaja de evaluar por separado la satisfacción con los distintos recursos sociales (recursos del equipo y los recursos del líder), permitiendo el desarrollo de medidas más específicas que incrementen la efectividad de las intervenciones y prácticas en post del desarrollo de organizaciones saludables y resilientes (Salanova et al., 2012)

Más allá de las implicaciones señaladas, es necesario destacar algunas limitaciones del presente estudio. Una de ellas concierne al método de recogida de datos. En efecto, a pesar de que la encuesta telefónica posee numerosas ventajas (e.g., mayor rapidez en la recolección y procesamiento de la información, menor invasión a la privacidad, acceso a áreas de gran inseguridad ciudadana), existen colectivos que no disponen en su hogar de telefonía fija. Este "error de cobertura" generaría una pérdida de representatividad de la información obtenida por medio de encuestas telefónicas (Díaz de Rada, 2001). Aunque existe evidencia que sugiere resultados similares utilizando encuestas telefónicas y presenciales (Galán, Rodríguez-Artalejo \& Zorrilla, 2004; Vogl, 2013), en el futuro sería provechoso replicar el estudio utilizando otras alternativas de recolección de datos (e.g., autoadministrado).

Adicionalmente, se ha cuestionado la validez de las encuestas telefónicas debido a la presencia de mayor aquiescencia, extremidad en las respuestas, y mayores respuestas socialmente deseables (Holbrook, Green \& Krosnich, 2003). Sin embargo, estudios recientes no hallaron este tipo de sesgos en encuestas telefónicas e, incluso, observaron una mayor presencia de sesgos de deseabilidad social en las encuestas autoadministradas (Díaz de Rada, 2011). Estos resultados reforzarían en principio la validez de las conclusiones obtenidas en este estudio, aunque son necesarias nuevas investigaciones.

Por último, sería conveniente realizar nuevos estudios que aporten evidencia adicional sobre la calidad psicométrica del CSRL_16, como la estabilidad temporal de las puntuaciones y la validez discriminante e incremental respecto de otras medidas, especialmente de escalas que evalúen recursos laborales. Prevemos realizar este estudio en los próximos meses. 
Aún con estas limitaciones el instrumento es confiable y aporta al desarrollo de organizaciones saludables; y se propone también como herramienta para futuros estudios sobre la satisfacción, el bienestar y la salud psicosocial de las personas en el trabajo.

\section{Conflicto de interés}

Los autores declaran que no existen ningún conflicto de interés relacionado con el contenido del artículo, o su publicación en Suma Psicológica.

\section{Referencias}

Aiken, L. (1985). Three coefficients for analyzing the reliability and validity of ratings. Educational and Psychological Measurement, 45, 131-142. http://dx.doi.org/10.1177/0013164485451012

Arias, B. (2008). Desarrollo de un ejemplo de análisis factorial confirmatorio con LISREL, AMOS y SAS. En M. A. Verdugo, M. Crespo, M. Badía \& B. Arias (Coords.), Metodología en la investigación sobre discapacidad. Introducción al uso de las ecuaciones estructurales. (pp. 75-120) Salamanca: INICO

Bakker, A. B., \& Demerouti, E. (2013). La teoría de las demandas y los recursos laborales. Journal of Work and Organizational Psychology, 29, 107-115. http://dx.doi.org/10.5093/tr2013a16

Bakker, A. B., \& Demerouti, E. (2018). Multiple levels in job demands-resources theory: Implications for employee well-being and performance. In E. Diener, S. Oishi \& L. Tay (Eds.), Handbook of well-being. (pp. 1-13) Salt Lake City, UT: DEF Publishers

Breevaart, K., \& Bakker, A.B. (2018). Daily job demands and employee work engagement: The role of daily transformational leadership behavior. Journal of Occupational Health Psychology, 23, 338-349. http://dx.doi.org/10.1037/ocp0000082

Cannon-Bowers, J. A., Bowers, C., \& Procci, K. (2010). Optimizing learning in surgical simulations: Guidelines from the science of learning and human performance. Surgical Clinics of North America, 90, 583-603. http://dx.doi.org/10.1016/j.suc.2010.02.006

Díaz de Rada, V. (2001). Problemas de cobertura en la encuesta telefónica. Reis: Revista Española de Investigaciones Sociológicas, 93, 133-164. http://dx.doi.org//10.2307/40184330

Díaz de Rada, V. (2011). Encuestas con encuestador y autoadministradas por internet. ¿Proporcionan resultados comparables? Reis: Revista Española de Investigaciones Sociológicas, 136, 49-90. http://dx.doi.org/10.5477/cis/reis.136.49

Dominguez-Lara, S. (2016). Evaluación de la confiabilidad del constructo mediante el Coeficiente $\mathrm{H}$ : Breve revisión conceptual y aplicaciones. Psychologia. Avances de la Disciplina, 10, 87-94. http://dx.doi.org/10.21500/19002386.2134

Duffy, R. D., \& Lent, R. W. (2009). Test of a social cognitive model of work satisfaction in teachers. Journal of Vocational Behavior, 75, 212-223. http://dx.doi.org/10.1016/j.jvb.2009.06.001

Durán, M. Extremera, N., Montalbán, F. M., \& Rey, L. (2005). Engagement y burnout en el ámbito docente: Análisis de sus relaciones con la satisfacción laboral y vital en una muestra de profesores. Revista de Psicología del Trabajo y de las Organizaciones, 21(1-2), 145-158.

Eignor, D. R. (2013). The standards for educational and psychological testing. En K. F. Geisinger, B. A. Bracken, J. F. Carlson, J.-I. C. Hansen, N. R. Kuncel, S. P. Reise, \& M. C. Rodriguez (Eds.), APA handbooks in psychology. APA handbook of testing and assessment in psychology (Vol. 1). Test theory and testing and assessment in industrial and organizational psychology (pp. 245-250). Washington, DC: American Psychological Association. http://dx.doi.org/10.1037/14047-013

Fabrigar, L. R., Wegener, D. T., MacCallum, R. C., \& Strahan, E. J. (1999). Evaluating the use of exploratory factor analysis in psychological research. Psychological Methods, 4, 272-299. http://dx.doi.org/10.1037//1082-989x.4.3.272
Furr, M. (2011). Scale construction and psychometrics for social and personality psychology. London: Sage.

Galán, I., Rodríguez-Artalejo, F., \& Zorrilla, B. (2004). Comparación entre encuestas telefónicas y encuestas «cara a cara» domiciliarias en la estimación de hábitos de salud y prácticas preventivas. Gaceta Sanitaria, 18, 440-450. http://dx.doi.org/10.1016/ s0213-9111(04)72031-2

George, D., \& Mallery, M. (2010). Using SPSS for Windows step by step: A simple guide and reference. Boston, MA: Allyn \& Bacon

Halbesleben, J. R. B., \& Demerouti, E. (2005). The construct validity of an alternative measure of burnout: Investigating the English translation of the Oldenburg Burnout Inventory. Work \& Stress, 19, 208-220. http://dx.doi.org/10.1080/02678370500340728

Hernández Sampieri, R., Fernández Collado, C., \& Baptista Lucio, P. (2010). Metodología de la investigación ( $5^{a}$ Ed.). México: McGraw-Hill.

Hoffmann, A., Stover, J., de la Iglesia, G. \& Fernández Liporace, M. (2013). Correlaciones policóricas y tetracóricas en estudios factoriales exploratorios y confirmatorios. Ciencias Psicológicas, 7, 151-164. http://dx.doi.org/10.1080/02678370500340728

Holbrook, A. L., Green, M. C., \& Krosnick, J. A. (2003). Telephone versus face-to-face interviewing of national probability samples with long questionnaires. Public Opinion Quarterly, 67, 79-125. http://dx.doi.org/10.1086/346010

Lent, R. W., \& Brown, S. D. (2008). Social cognitive career theory and subjective well-being in the context of work. Journal of Career Assessment, 16, 6-21. http://dx.doi.org/10.1177/1069072707305769

$\mathrm{Li}, \mathrm{C}$. (2016). Confirmatory factor analysis with ordinal data: Comparing robust maximum likelihood and diagonally weighted least squares. Behavior Research Methods, 48, 936-949. http:// dx.doi.org/10.3758/s13428-015-0619-7

Llorens, S., Salanova, M., \& Ventura, M. (2009). El tecnoestrés: Un problema de nuestros días. Aprende Rh, 26, 96-102.

Lloret, S., Ferreres, A., Hernández, A., \& Tomás, I. (2017). El análisis factorial exploratorio de los ítems: análisis guiado según los datos empíricos y el software. Anales de Psicología 33, 417432. http://dx.doi.org/10.6018/analesps.33.2.270211

Lloret-Segura, S., Ferreres-Traver, A., Hernández-Baeza, A., \& Tomás-Marco, I. (2014). El análisis factorial exploratorio de los ítems: una guía práctica, revisada y actualizada. Anales de Psicología, 30, 1151-1169. http://dx.doi.org/10.6018/analesps.30.3.199361

Lorenzo-Seva, U. (1999). Promin: A method for oblique factor rotation. Multivariate Behavioral Research, 34, 347-365. http:// dx.doi.org/10.1207/S15327906MBR3403_3

Medrano, L. A., Pérez, E., \& Fernández, A. (2019). Construcción y adaptación de test psicométricos. En L. A. Medrano \& E. Pérez (Eds.), Manual de psicometría y evaluación psicológica (pp. 89-100): Córdoba: Editorial Brujas.

Merino, C., \& Livia, J. (2009). Intervalos de confianza asimétricos para el índice de validez de contenido: Un programa Visual Basic para la V de Aiken. Anales de Psicología, 25, 169-171.

Penfield, R., \& Giacobbi, P. Jr. (2004) Applying a score confidence interval to Aiken's item content-relevance index. Measurement in Physical Education and Exercise Science, 8, 213-225. http:// dx.doi.org/10.1207/s15327841mpee0804_3

Pérez, E., \& Medrano, L. A. (2010). Análisis factorial exploratorio: Bases conceptuales y metodológicas. Revista Argentina de Ciencias del Comportamiento, 2, 58-66.

Rivas Moya, T. (1999). Comparación de procedimientos para analizar estructuras factoriales en muestras independientes. Revista Electrónica de Metodología Aplicada, 4, 19-43.

Rodríguez Ayán, M., \& Ruiz Díaz, M. (2008). Atenuación de la asimetría y de la curtosis de las puntuaciones observadas mediante transformaciones de variables: incidencia sobre la estructura factorial. Psicológica, 29, 205-227.

Rothmann, S. (2008). Job satisfaction, occupational stress, burnout and work engagement as components of work-related wellbeing. SA Journal of Industrial Psychology, 34, 11-16. 
Salanova, M, \& Schaufeli, W. B. (2004). El engagement de los empleados: Un reto emergente para la dirección de los recursos humanos. Estudios financieros, 261, 109-138.

Salanova, M. (2008). Organizaciones saludables: Una aproximación desde la Psicología Positiva. En C. Vázquez \& G. Hervás (Eds.), Psicología positiva aplicada (pp. 403-427). Bilbao: Desclée de Brower.

Salanova, M., Llorens, S., \& M. Martínez, I. (2019). Organizaciones Saludables. Una mirada desde la psicología positiva (1 ed.). España: Aranzadi.

Salanova, M., Llorens, S., Cifre, E., \& Martínez, I. (2006b). Metodología RED-WoNT. En Perspectivas de intervención en riesgos psicosociales (pp. 131-153). Barcelona: Foment del Treball Nacional.

Salanova, M., Llorens, S., Cifre, E., \& Martínez, I. M. (2012). We need a hero! Toward a validation of the Healthy and Resilient Organization (HERO) Model. Group \& Organization Management, 37, 785-822. http://dx.doi.org/10.1177/1059601112470405

Salanova, M., Martínez, I. M., \& Llorens, S. (2014). Una mirada más "positiva" a la salud ocupacional desde la Psicología Organizacional Positiva en tiempos de crisis: Aportaciones desde el equipo de investigación WONT. Papeles del Psicólogo, 35, 22-30.

Sánchez-Manzanares, M., Rico, R., \& Gil, F. (2008). Designing organizations: Does expertise matter? Journal of Business and Psychology, 23, 87-101. http://dx.doi.org/10.1007/s10869-0089076-y

Schaufeli, W. B., \& Taris, T. W. (2014). A critical review of the Job Demands-Resources Model: Implications for improving work and health. En G. Bauer \& O. Hämmig (Eds.), Bridging occupational, organizational and public health (pp. 43-68). Dordrecht, the Netherlands: Springer. http://dx.doi.org/10.1007/97894-007-5640-3_4

Schaufeli, W. B., Taris, T. W., \& van Rhenen, W. (2008). Workaholism, burnout, and work engagement: Three of a kind or three different kinds of employee well-being? Applied Psychology: An International Review, 57, 173-203. http://dx.doi.org/10.1111/ j.1464-0597.2007.00285.x

Schaufeli, W., Leiter, M., \& Maslach, C. (2009). Burnout: 35 years of research and practice. Career Development International, 14, 204-220. http://dx.doi.org/10.1108/13620430910966406

Schaufeli, W., Leiter, M., Maslach, C., \& Jackson, S. (1996). Maslach Burnout Inventory - General Survey. En C. Maslach, S. Jackson \& M. Leiter (Eds.), The Maslach Burnout Inventory-Test Manual ( $3^{\circ}$ Ed.). Palo Alto, CA: Consulting Psychologists Press.

Schaufeli, W., Salanova, M., González-Romá, V., \& Bakker, A. (2002). The measurement of burnout and engagement: A confirmatory factor analytic approach. Journal of Happiness Studies, 3, 71-92. http://dx.doi.org/10.1023/A:1015630930326
Spontón, C., Medrano, L. A., Maffei, L., Spontón, M., \& Castellano, E. (2012). Validación psicométrica del cuestionario de engagement UWES a la población de trabajadores de Córdoba, Argentina. Liberabit, 18, 147-154.

Spontón, C., Trógolo, M., Medrano, L. A., \& Castellano, E. (2019). Medición del burnout: Estructura factorial, validez y confiabilidad en trabajadores argentinos. Interdisciplinaria, 36(1), 87-103.

Stacey, N., Ellwood, P., Bradbrook, S., Reynolds, J., Tavetz, J., Williams, H., \& Lye D. (2018). Foresight on new and emerging occupational safety and health risks associated digitalisation by 2025. Luxembourg: European Agency for Safety and Health at Work. http://dx.doi.org/10.2802/515834

Tabachnick, B., \& Fidell, L. (2001). Using Multivariate Statistics $\left(4^{\text {th }}\right.$ Ed). Boston, Massachusetts: Allyn \& Bacon.

Thompson, C. A., \& Prottas, D. J. (2006). Relationships among organizational family support, job autonomy, perceived control, and employee well-being. Journal of Occupational Health Psychology, 11,100-118. http://dx.doi.org/10.1037/1076-8998.10.4. 100

Timmerman, M. E., \& Lorenzo-Seva, U. (2011). Dimensionality assessment of ordered polytomous items with parallel analysis. Psychological Methods, 16, 209-220. http://dx.doi.org/10.1037/ a0023353

Tripiana, J., \& Llorens, S. (2015). Fomentando empleados engaged: El rol del líder y de la autoeficacia. Anales de Psicología, 31, 636-644. http://dx.doi.org/10.6018/analesps.31.2.179561

Trógolo, M., Pereyra, A. P., \& Spontón, C. (2013). Impacto de diferentes estilos de liderazgo sobre el engagement y burnout: Evidencia en una muestra de trabajadores argentinos. Ciencia \& trabajo, 15, 152-157. http://dx.doi.org/10.4067/S071824492013000300008

Vogl, S. (2013). Telephone versus face-to-face interviews: Mode effect on semistructured interviews with children. Sociological Methodology, 43, 133-177. http://dx.doi.org/10.1177/0081175012465967

Warr, P. (1990). The measurement of well-being and other aspects of mental health. Journal of Occupational Psychology, 63, 193-210. http://dx.doi.org/10.1111/j.2044-8325.1990.tb00521.x

Yu. C., \& Muthen. B. (2002). Evaluation of model fit indices for latent variable models with categorical and continuous outcomes. Paper presented at the annual meeting of the American Educational Research Association. New Orleans. LA.

Yukl, G., Gordon, A., \& Taber, T. (2002). A hierarchical taxonomy of leadership behavior: integrating a half century of behavior research. Journal of Leadership \& Organizational Studies, 9, 15-32. http://dx.doi.org/10.1177/107179190200900102 